\title{
Estado nutricional e autopercepção da imagem corporal de idosas de uma Universidade Aberta da Terceira Idade
}

\author{
Nutritional status and self-perceived body image of elderly women at an Open University of the \\ Third Age
}

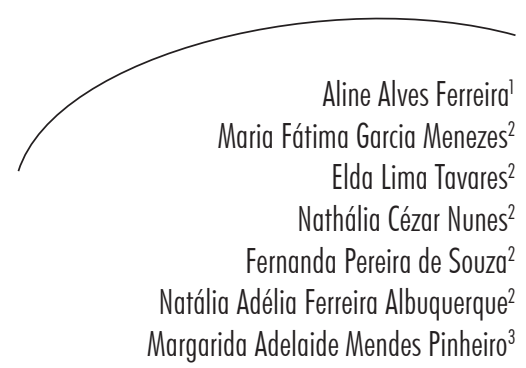

\section{Resumo}

O objetivo foi avaliar a associação entre o estado nutricional e a insatisfação da autoimagem corporal de idosas matriculadas no curso Alimentação, Nutrição e Terceira Idade da UnATI/UERJ. Realizou-se estudo seccional, com entrevista semiestruturada com todas as idosas ( $\geq 60$ anos), obtendo-se informações referentes à percepção da imagem corporal (escala de nove silhuetas de Stunkard), indicadores socioeconômicos, de saúde, demográficos e cálculo do índice de massa corporal (IMC). Os dados foram analisados por meio de teste qui-quadrado e a regressão linear simples (nível de significância $=5 \%$ ). Verificou-se que 50,0\% eram eutróficas; $6,0 \%$ se encontravam na categoria de baixo-peso e 44,0\% apresentavam sobrepeso $(\mathrm{p}=0,425)$. Quanto à percepção da imagem corporal, 74,0\% (n=37;p=0,0049) estavam insatisfeitas com o corpo, principalmente pelo excesso $(89,2 \%)$. Das pessoas que manifestaram insatisfação por baixo-peso, a maioria era eutrófica. Já entre as insatisfeitas por excesso, 30,2\% não apresentavam excesso de peso. As silhuetas 2 e 3 foram apontadas como ideais por metade das entrevistadas, ainda que a autopercepção de 62,0\% das idosas tenham sido as silhuetas 4 e 5 . A barriga e membros, principalmente inferiores, foram as partes do corpo que as idosas menos gostavam (78,0\%). A classificação na escala das silhuetas da imagem real aumenta conforme aumenta o IMC $(\mathrm{p}<0,001)$. Em relação à imagem ideal ocorre o oposto $(\mathrm{p}<0,001)$. Indivíduos com IMC elevado tendem a ser mais insatisfeitos com sua imagem corporal e essa insatisfação pode estar relacionada ao excesso de peso. Esse fato merece mais aprofundamento sobre os determinantes e os significados desta insatisfação na complexa relação corpo, beleza, saúde e envelhecimento.

\footnotetext{
Departamento de Nutrição Social e Aplicada, Instituto de Nutrição Josué de Castro. Universidade do Federal do Rio de Janeiro. Rio de Janeiro, RJ, Brasil.

2 Departamento de Nutrição Social, Instituto de Nutrição. Universidade do Estado do Rio de Janeiro. Rio de Janeiro, RJ, Brasil.

3 Escola Superior de Biotecnologia. Universidade Católica Portuguesa. Porto, Portugal.
}

\author{
Correspondência / Correspondence \\ Aline Alves Ferreira \\ Instituto de Nutrição Josué de Castro - UFRJ \\ Av. Carlos Chagas Filho, 373 - Ed. do Centro de Ciências da Saúde \\ Bloco J, sala $10,2^{\circ}$ andar - Cidade Universitária - \\ 21941-902 Rio de Janeiro, RJ, Brasil \\ E-mail: alineaf@nutricao.ufri.br
}

Palavras-chave: Imagem Corporal. Estado Nutricional. Idoso. 


\section{Abstract}

The study aimed to estimate the association between nutritional status and body selfimage dissatisfaction in elderly women attending the Food, Nutrition and Elderly Course at UnATI/UERJ. A cross-sectional study was conducted, with semi-structured interviews with all elderly ( $\geq 60$ years), obtaining information regarding body image perception (scale of nine Stunkard silhouettes), socioeconomic, health and demographic indicators, and BMI. The data were analyzed using the Chi-square test and linear regression (significance level $=5 \%$ ). It was found that $50.0 \%$ had normal weight; $6.0 \%$ were in the category of underweight; and $44.0 \%$ were overweight $(\mathrm{p}=0.425)$. Regarding the perception of body image, $74.0 \%(n=37 ; \mathrm{p}=0.0049)$ were dissatisfied with the body, mainly by excess $(89.2 \%)$. Of those who expressed dissatisfaction with low weight, most were eutrophic. On the other hand, between the ones dissatisfied by excess, $30.2 \%$ are not overweight. Silhouettes 2 and 3 were identified as ideal for half of the respondents, although the self-perception of $62.0 \%$ of the elderly had been the silhouettes 4 and 5. The belly and limbs, especially lower body parts are the least liked by the older (78.0\%). The silhouettes rating scale of the actual image increases as BMI $(\mathrm{p}<0.001)$. The opposite occurs regarding the ideal image $(\mathrm{p}<0.001)$. Individuals with high BMI tend to be more dissatisfied with their body image and this may be related to weight excess. This fact deserves deeper understanding of the determinants and meanings of this complex relationship dissatisfaction between body, beauty, health and aging.
Key words: Body Image. Nutritional Status. Elderly.

\section{INTRODUÇÃO}

O processo do envelhecimento envolve diferentes aspectos, incluindo modificações fisiológicas, socioeconômicas, alterações de estruturas familiares, além de demandas por políticas públicas e redistribuição de recursos na sociedade. $^{1}$

Inserido nesse processo de envelhecimento, o corpo também se modifica, especialmente nas mulheres. ${ }^{2,3}$ A quantidade e a distribuição do tecido adiposo e de massa muscular são alteradas, resultando comumente em aumento do tecido adiposo, especialmente até os 75 anos de idade. Paralelamente, há uma perda gradual e natural de massa óssea, massa muscular-esquelética e água corporal total, resultando em diminuição na estatura e outras modificações corpóreas. ${ }^{2,4}$

O modo com que um indivíduo percebe seu corpo é influenciado por aspectos pessoais pregressos, aspectos econômicos, religiosos, pertencimento à sociedade, além de outras intervenientes sociais e culturais. ${ }^{3,5}$ Desse modo, o corpo é socialmente construído, podendo ser considerado o principal elo de ligação do indivíduo com a sociedade. ${ }^{5}$

A busca pelo corpo perfeito e rejuvenescido, com forte rejeição às marcas naturais do envelhecimento, caracteriza a sociedade contemporânea. Hábitos alimentares inadequados, com dietas de restrições calóricas severas, tratamentos medicamentosos e cirúrgicos, além de exercícios físicos vigorosos, configuram o cenário moderno da "indústria da beleza", que também atinge o público mais velho. ${ }^{6}$

As idosas têm apresentado preocupações e insatisfações com a imagem corporal, buscando uma melhor aparência física. Há uma influência constante da mídia e da sociedade sob essa autopercepção e satisfação do corpo. ${ }^{2,6,7}$ Não raro, relatos de insatisfação com o corpo envelhecido evidenciados na literatura, são relacionados com as condições socioeconômicas e o estado nutricional. ${ }^{8}$ 
A percepção da aparência corporal, importante componente de identidade pessoal, é uma construção multidimensional que representa como a pessoa percebe seu próprio corpo. A idade, gênero, índice de massa corporal (IMC) e condição socioeconômica comumente estão associados nesse processo. ${ }^{7,9}$ A avaliação dessa imagem corporal por meio de escalas de silhuetas pode ser um instrumento importante na avaliação do estado nutricional, uma vez que explora outros componentes e dimensões. ${ }^{10}$

A discussão sobre percepção da imagem corporal em idosos contribui para a utilização de diferentes indicadores que avaliam o bem-estar dessa população. Assim, o objetivo deste estudo foi avaliar a associação entre o estado nutricional e a insatisfação da autoimagem corporal de idosas matriculadas no curso Alimentação, Nutrição e Terceira Idade da UnATI/UERJ.

\section{METODOLOGIA}

A Universidade Aberta da Terceira Idade (UnATI) foi criada em 1993 e, desde então, mantém parceria com o Departamento de Nutrição Social do Instituto de Nutrição (INU) da Universidade do Estado do Rio de Janeiro (UERJ). O Projeto de Extensão Nutrição e Terceira Idade articula extensão, ensino e pesquisa, com diferentes atividades direcionadas aos idosos, dentre elas o curso Alimentação, Nutrição e Terceira Idade.

O curso ocorre anualmente em aulas semanais (1h50min) e oferece 50 vagas em duas turmas, por meio de sorteio. São utilizadas diferentes estratégias metodológicas, a fim de problematizar a realidade e estimular a parceria e o diálogo. ${ }^{11} \mathrm{~A}$ atividade inicial do curso consiste de entrevista individual, objetivando conhecer o perfil de saúde, nutrição e alimentação do grupo, fornecendo subsídios para o planejamento das aulas ao longo do ano.
Nesse contexto, em fevereiro de 2012, foi realizada entrevista semiestruturada contendo informações referentes a alimentação, nutrição, imagem corporal, além de aspectos sociodemográficos e condições de saúde autorreferidas. O presente estudo foi realizado com todas as idosas inscritas no curso $(n=50)$, com idade superior a 60 anos, sem técnicas específicas de amostragem. Os dados foram coligidos numa única semana, na parte da manhã, no Laboratório Interdisciplinar de Avaliação Nutricional (LIAN) do INU/UERJ.

Para avaliação do estado nutricional, foram mensuradas as variáveis antropométricas "estatura" e "massa corporal", conforme técnicas preconizadas por Lohman et al. ${ }^{12} \mathrm{Com}$ base nestas aferições, foi calculado o Índice de Massa Corporal (IMC), sendo a classificação baseada nos pontos de corte preconizados por Lipschitz. ${ }^{13}$ A massa corporal foi medida utilizando-se uma balança eletrônica marca Filizola, capacidade $180 \mathrm{~kg}$, precisão de 0,1 kg (Indústria Filizola S/A, Brasil). A estatura foi aferida com um estadiômetro vertical da marca Alturaexata (Alturaexata Ltda., Brasil.), com precisão de $1 \mathrm{~mm}$.

A percepção da imagem corporal considerada real e ideal foi autorreferida por meio da escala de nove silhuetas, proposta por Stunkard et al., ${ }^{14}$ que classifica desde a magreza (silhueta 1) até a obesidade severa (silhueta 9), conforme a figura 1. Para avaliação da percepção da imagem corporal, obteve-se a diferença entre a aparência corporal real e a aparência corporal ideal. Se a variação fosse igual a zero, classificava-se a idosa como satisfeita; e se diferente de zero, classificava-se como insatisfeita. Caso a diferença fosse positiva, considerou-se insatisfação pelo excesso de peso e, quando negativa, insatisfação pela magreza. Também foram aplicadas perguntas abertas referentes a partes do corpo que mais gostavam e menos gostavam. 

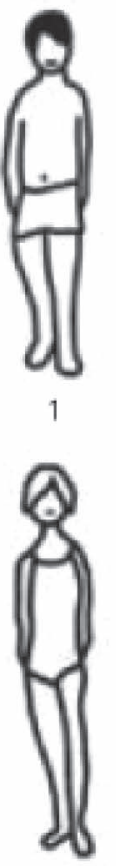

1

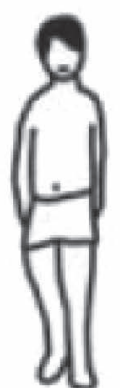

2

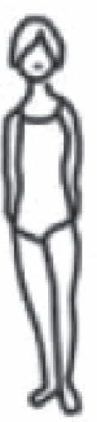

2

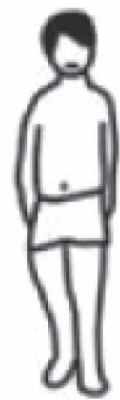

3

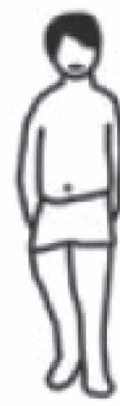

4

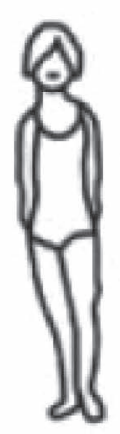

3

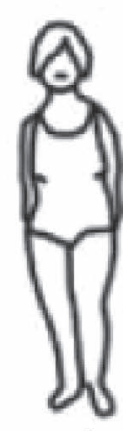

4

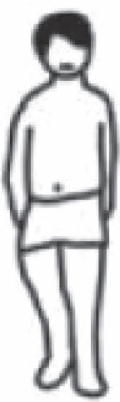

5

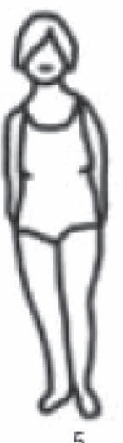

5

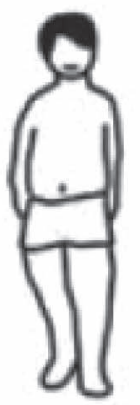

6

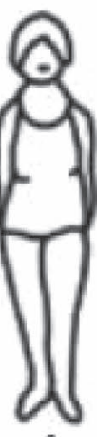

6

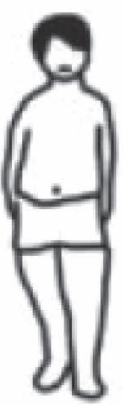

7

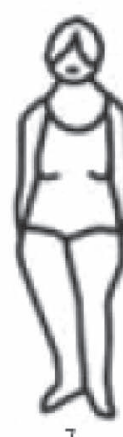

7
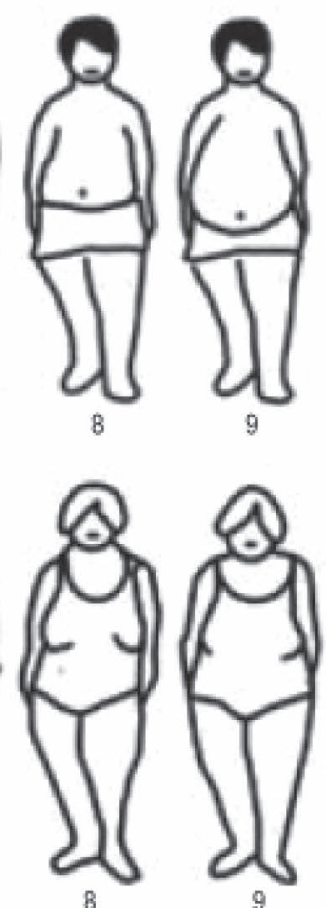

Figura 1. Desenhos das silhuetas baseadas na escala proposta por Stunkard e colaboradores. ${ }^{14}$ Rio de Janeiro-RJ, 2012.

Foram verificadas a satisfação e a insatisfação da imagem corporal em relação a idade, escolaridade, arranjo domiciliar, condição econômica e alteração em saúde. Os dados foram analisados por meio de teste qui-quadrado (nível de significância $=5 \%$ ).

Após o cumprimento dos pressupostos paramétricos, foi realizada a regressão linear simples. A variável resposta foi o IMC, e as variáveis independentes foram as condições socioeconômicas, demográficas e as condições de saúde autorreferidas, além da imagem corporal ideal e a imagem corporal real (inseridas em modelos separados), com nível de significância de 5\%. As análises foram feitas por meio do software SPSS - Statistical Package for the Social Sciences for Windows, versão 19.0.
O projeto foi aprovado pelo Comitê de Ética em Pesquisa da Universidade do Estado do Rio de Janeiro (CEP/UERJ no 029.3.2005) e Comitê de Ética em Pesquisa da Secretaria Municipal de Saúde (CEP SMS/RJ no 172A/2006), de acordo com a Resolução CNS no 196/96, do Conselho Nacional de Saúde. As idosas, após receberem informações sobre os procedimentos para coleta de dados, assinaram o Termo de Consentimento Livre e Esclarecido.

\section{RESULTADOS}

Foram analisados 50 indivíduos, todos do sexo feminino, participantes no curso Alimentação, Nutrição e Terceira Idade, no ano de 2012. A idade média foi de 71,02 anos 
$(\mathrm{dp}=62,35)$, sendo a idade mínima igual a 61 anos e a máxima, 87 anos. Verificou-se que houve predomínio de idosas que moravam sozinhas $(44,0 \%)$, com renda mensal inferior a dois salários mínimos $(52,2 \%)$ e ensino médio completo (42,0\%). Por outro lado, $34,8 \%$ possuíam renda mensal superior a seis salários mínimos, nenhum indivíduo era analfabeto e $13(26,0 \%)$ possuíam nível superior completo ou pós-graduação. Autorreferenciaram, em sua maior parte, um bom estado de saúde (46,0\%).

A figura 2 evidencia a distribuição do estado nutricional em relação ao IMC, de acordo com a satisfação e insatisfação corporal. Em relação à classificação do estado nutricional de acordo com o IMC, verificou-se que 50,0\% eram eutróficas, $6,0 \%$ se encontravam na categoria de baixo-peso e $44,0 \%$ apresentavam sobrepeso $(p=0,425)$. A maioria das idosas estava insatisfeita com o corpo $(74,0 \%)$, quer seja pelo excesso ou baixopeso. Dentre as insatisfeitas, $89,2 \%$ apresentavam insatisfação pelo excesso de peso. As idosas que manifestaram insatisfação por baixo-peso eram, em sua maioria, eutróficas. Por outro lado, as insatisfeitas por excesso de massa corporal $(30,2 \%)$ não apresentaram excesso de peso, de acordo com o IMC.

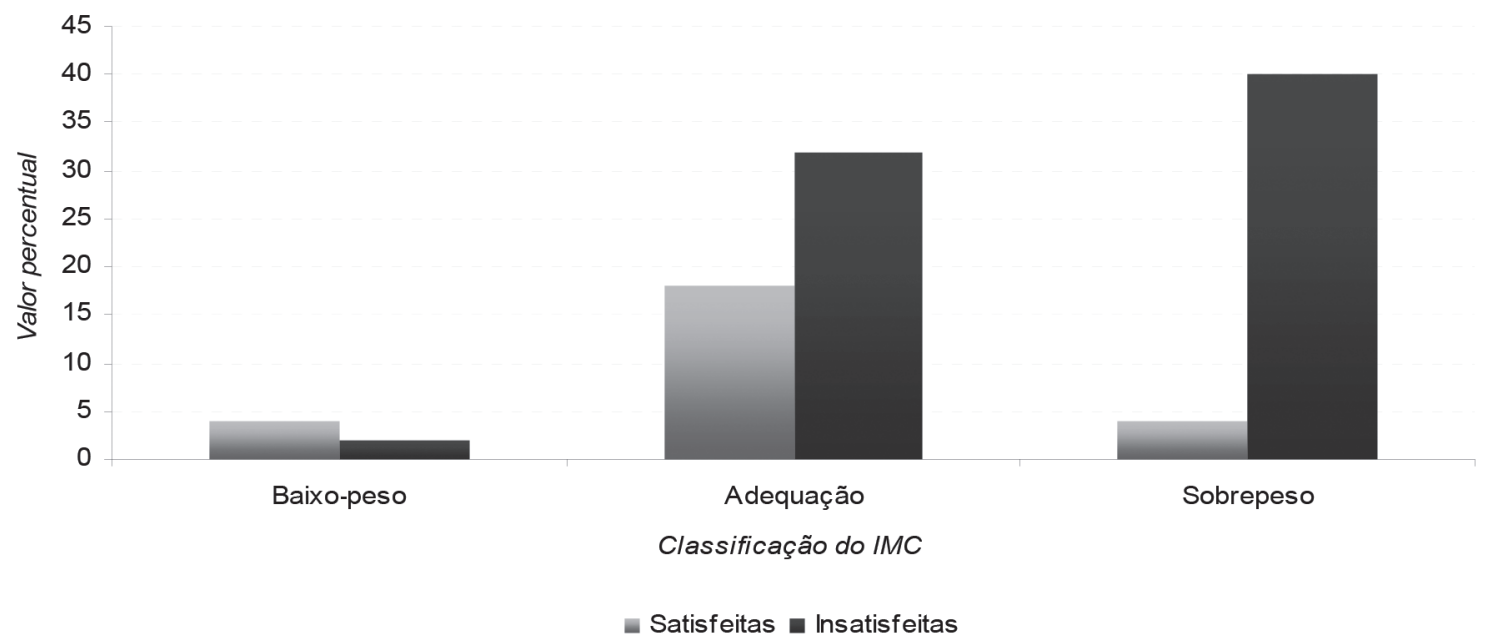

Figura 2. Distribuição percentual da classificação do estado nutricional, segundo o IMC. Rio de Janeiro-RJ, 2012.

Em relação à autopercepção da imagem corporal, a silhueta 3 foi apontada como imagem ideal por quase metade das entrevistadas $(40,0 \%)$. Em contrapartida, a autoimagem que correspondia à realidade da maior parte das idosas (imagem real) foram as silhuetas 4 e 5
$(62,0 \%)$, conforme mostrado na figura 3. O abdômen e membros, principalmente inferiores, foram as partes do corpo que as idosas menos gostavam (78,0\%). A parte do corpo que mais gostavam foi o rosto, especialmente os olhos $(24,0 \%)$. 


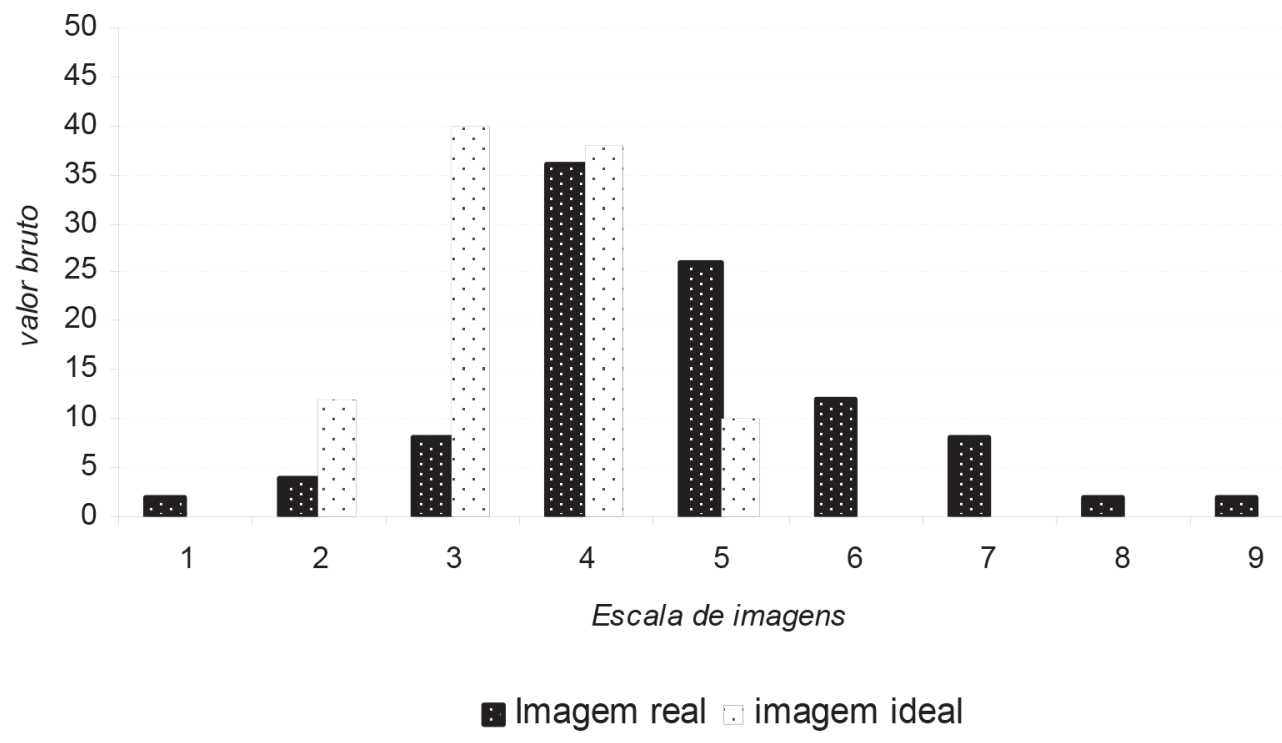

Figura 3. Distribuição percentual da autoclassificação da imagem corporal real e ideal. Rio de JaneiroRJ, 2012.

Quando relacionada à satisfação e insatisfação do corpo em relação a algumas características socioeconômicas, demográficas e de saúde, percebeu-se que a maior parte das idosas que apresentava alguma insatisfação corporal tinha menos de 70 anos de idade, ensino médio completo (40,0\%), recebia menos que quatro salários mínimos mensais $(37,0 \%)$ e moravam só $(34,0 \%)$. A diferença entre as idosas satisfeitas e insatisfeitas com o corpo foi estatisticamente significativa ( $\mathrm{p}$-valor $<0,05$ ) para as idosas com mais de 70 anos e ensino médio completo. Essa diferença também foi evidenciada independentemente do nível de renda e arranjo domiciliar. Já 30,0\% das idosas insatisfeitas apresentavam estado de saúde regular ou bom. A insatisfação com o corpo somente esteve associada à autoclassificação de saúde regular ( $\mathrm{p}$-valor $<0,001)$, segundo a tabela 1. 
Tabela 1. Frequência absoluta e relativa das idosas, segundo a satisfação com a imagem corporal e condições socioeconômicas . Rio de Janeiro-RJ, 2012.

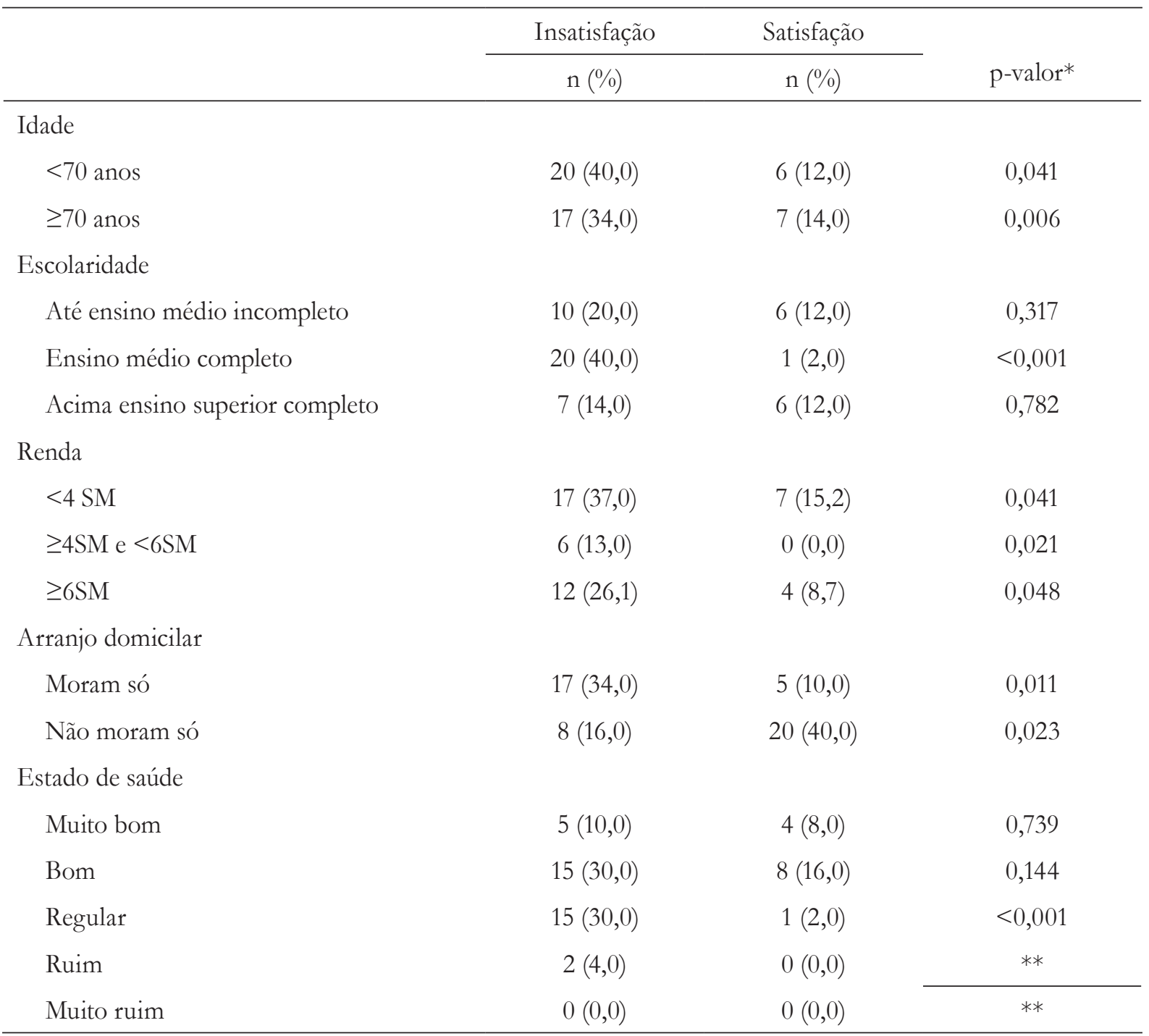

*Teste qui-quadrado entre os percentuais de idosos satisfeitos e insatisfeitos com o corpo; **não aplicável (n=0).

Após a modelagem, apenas a imagem ideal e a real permaneceram no modelo. A classificação na escala das silhuetas da imagem real aumenta conforme aumenta o IMC ( $\mathrm{p}<0,001)$. Em relação à imagem ideal, ocorre o oposto: conforme aumenta a categoria do IMC, diminui a classificação da imagem ideal desejada pelas idosas $(\mathrm{p}<0,001)$. Ou seja, as idosas que possuem IMC maior tendem a almejar uma imagem corporal mais magra quando comparadas às idosas que apresentam IMC menor. Assim, indivíduos com IMC elevado tendem a ser mais insatisfeitos com sua imagem corporal e essa insatisfação pode estar relacionada ao excesso de peso ou maior IMC (figura 4). 

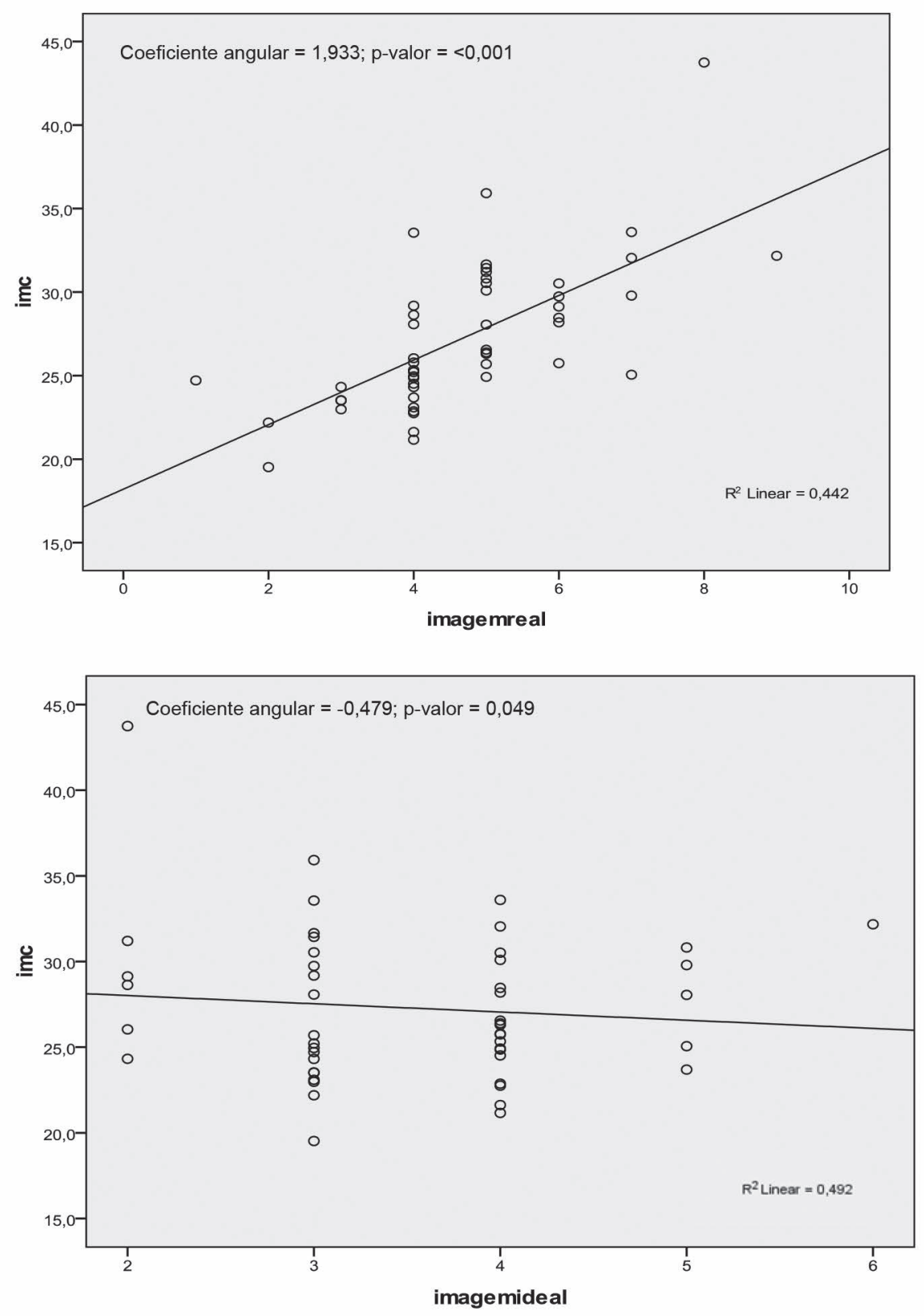

Figura 4. Relação das imagens real e ideal com relação ao IMC. Rio de Janeiro-RJ, 2012.

\section{DISCUSSÃO}

O perfil nutricional em indivíduos idosos também vem se modificando nas últimas décadas. O excesso de peso nesse segmento populacional tem aumentado, independentemente das classes socioeconômicas inferiores. ${ }^{15}$ De acordo com a Pesquisa Nacional sobre Vigilância de Fatores de Risco e Proteção para Doenças Crônicas por Inquérito Telefônico (Vigitel) de 2012, ${ }^{16}$
$59,1 \%$ das mulheres com mais de 65 anos de idade apresentam excesso de peso. Os achados do presente estudo, $44,0 \%$ de excesso de peso, são inferiores se comparados a esses valores ou a outros inquéritos nacionais (59,5\% para mulheres entre 65 e 74 anos). ${ }^{15}$

Estudos ressaltam que, quanto maior a idade, menor a tendência de prevalência de sobrepeso e obesidade entre idosos., ${ }^{4,13,15,17,18}$ 
Modificações importantes na distribuição de massa muscular e de gordura corporal ocorrem com o envelhecimento, havendo diminuição da massa corpórea. ${ }^{2,4}$ Esse cenário tende a ser maior em mulheres. ${ }^{18}$ Silveira e colaboradores ${ }^{17}$ evidenciaram que as frequências de obesidade decresceram de forma homogênea com o avançar das faixas de idade em idosos de Pelotas-RS.

Tanto o baixo-peso quanto a obesidade estão associados a uma maior morbimortalidade em idosos. O baixo-peso, ainda que em pequenas proporções no presente estudo (6,0\%), merece atenção, pois pode estar relacionado a complicações como: infecções intermitentes, problemas respiratórios, deficiências nutricionais e outras consequências relacionadas à função gastrointestinal. ${ }^{18}$

Tão importante quanto o estado nutricional é a relação que o idoso mantém com seu corpo e com seu bem-estar. A alteração da distribuição de gordura corpórea e outras mudanças do corpo relacionadas à idade são comuns: aparecimento de rugas e sinais senis, além da diminuição da aptidão física, com perda de massa muscular. Essas mudanças afetam diretamente a satisfação com o corpo, especialmente entre as mulheres. ${ }^{2}$

O corpo, para Le Breton, ${ }^{5}$ "é uma construção simbólica, não uma realidade em si [...]. Ele nunca é um dado indiscutível, mas o efeito de uma construção social e cultural". Em uma perspectiva individualista atual, o corpo é visto como separado do indivíduo, é o "recinto do sujeito, o lugar de seu limite e de sua liberdade, o objeto privilegiado de uma fabricação e de uma vontade de domínio". ${ }^{5}$

Segundo Goldenberg, ${ }^{19}$ o corpo no Brasil, em comparação com outras culturas, é um verdadeiro capital, uma riqueza. Um corpo jovem, magro, em boa forma, sexy, conquistado à custa de muito investimento financeiro, trabalho e sacrifício material e corporal. Na pesquisa Hapiness Brasil, que busca compreender a "cultura da felicidade", Ramos $^{20}$ destaca como os cuidados com o corpo e a produção da aparência estão no centro de situações e momentos de felicidade. $\mathrm{O}$ autor afirma que no discurso sobre o que falta para as mulheres serem mais felizes com o corpo encontra-se a referência de "mulheres, de diferentes idades, que estão em permanente luta contra o envelhecimento e a gordura, e que estão sempre incomodadas com mínimas imperfeições, acreditando que o corpo idealizado as faria mais felizes". ${ }^{20}$

O envelhecimento pode, portanto, ser experimentado como um momento de perda desse capital e, em consequência, perda de espaço, valor e prestígio social. Nesse sentido, as idosas do curso Alimentação, Nutrição e Terceira Idade apresentaram grande insatisfação com o corpo. O excesso de peso pode ser a principal explicação. Apesar de existirem poucos estudos nacionais que abordem a relação de satisfação e insatisfação corpórea em idosos, alguns achados foram semelhantes. ${ }^{10,21,22} \mathrm{Em}$ estudo com idosas do Sul do país, a prevalência de insatisfação foi similar ao achado no presente estudo (72,6\%). ${ }^{21}$ Já no estudo de Tribess e colaboradores, ${ }^{10}$ com idosas de Jequié-BA, 54,0\% demonstram insatisfação com a aparência do corpo.

A compreensão de aspectos relacionados à maior ou menor satisfação com a imagem corporal no idoso é fundamental para ações mais direcionadas a esse público. Entre as idosas do curso, houve diferença significativa entre a satisfação e insatisfação da imagem corporal e renda, além do arranjo familiar, da idade e nas concluintes do ensino médio que possuíam saúde autorrelatada como regular. A associação da condição socioeconômica com a percepção da imagem corporal é documentada em alguns estudos com idosas, ${ }^{22-24}$ mas não a escolaridade diretamente. Isso ocorre porque, em sua maioria, os anos de estudos das populações idosas são poucos ou nulos, ${ }^{10}$ ainda que a escolaridade das idosas que frequentavam o curso seja bastante superior à média local e nacional. ${ }^{25}$

A renda elevada pode ser considerada como facilitadora de diversos procedimentos para alterar o corpo de modo positivo, porém não tem sido evidenciada como fator predominante na satisfação corporal. ${ }^{8}$ As idosas da UnATI/UERJ também apresentaram perfil semelhante, uma vez que a diferença entre satisfação e insatisfação com o corpo ocorre de modo significativo, 
independentemente da renda. McLaren \& $\mathrm{Kuh}^{24}$ evidenciam como a insatisfação com o corpo pode ocorrer em indivíduos idosos de classes sociais mais elevadas também. A motivação para um corpo saudável e magro é um reflexo das normas pelas quais as mulheres são constantemente julgadas na sociedade ocidental., 82,24

Em relação à autopercepção da saúde, 46\% das idosas relataram ter um bom estado de saúde. Panorama semelhante foi encontrado nos idosos do país (45,5\%), ainda que 77,4\% tenham declarado ter doenças crônicas, incluindo obesidade..$^{25}$ Nesse sentido, ainda que boa parte as idosas tenha alegado ter um estado de saúde muito bom ou bom, a diferença na insatisfação corporal esteve presente apenas nas que autoclassificaram sua condição de saúde como "regular". Apesar do aumento de obesidade e outras doenças crônicas em idosos ao longo dos últimos anos, tem havido melhora considerável na autoavaliação do estado de saúde, especialmente no Sudeste. ${ }^{26}$

A estrutura familiar foi indiferente em relação à satisfação corporal das idosas. A relação do corpo com variáveis relacionadas à estrutura familiar foi documentada por Harris, ${ }^{27}$ que evidencia que um apoio psicossocial e familiar, independentemente do número de pessoas que residem com os idosos, apresenta efeitos muito maiores na autopercepção da aparência física, prática de atividade física e investimento em cuidados com a saúde. Outros autores, como Morais e colaboradores, ${ }^{28}$ têm ressaltado o efeito independente e significativo que um envelhecimento bem-sucedido apresenta em relação à estrutura familiar e amizades.

Neste estudo, idade também foi fator independentena satisfação dasidosas com o corpo. Tanto as idosas com mais de 70 anos de idade, ou menos, apresentaram diferenças significantes em relação à satisfação e insatisfação corporal. Alguns autores têm alertado que a felicidade e um comportamento positivo perante a vida são bem mais importantes na autopercepção da imagem corporal que a idade. ${ }^{9}, 29$ Não que uma idade mais avançada afete algumas facetas do corpo (por exemplo, desejo de emagrecer), mas pode não afetar tão intensamente outras (por exemplo, tamanho do corpo aceitável). ${ }^{2}$ Em um grupo de mulheres norte-americanas com diferentes idades, as insatisfações com distintas partes do corpo foram semelhantes entre as mais jovens e com mais idade. ${ }^{29}$

Assim, é necessário refletir a "força" que as questões culturais assumem para sua estruturação e significação da autopercepção corpórea. Não apenas os aspectos nutricionais, relativos à quantidade de alimentos e nutrientes, mas também os aspectos que norteiam a comida, que incluem com quem, como, onde e de que forma devem ser considerados. Comida e comensalidade estão juntas: comprar, planejar, preparar e comer agregam aspectos na estrutura alimentar. Por outro lado, a banalização, a desritualização e a desmotivação podem caminhar paralelamente nesse processo. Assim, Hernández \& Arnáiz ${ }^{30}$ enfatizam que é comum, em idosas que vivem só, a tendência a negligenciar a dieta e o próprio estado nutricional, apresentando padrões que denominam "comportamentos anárquicos", que incluem alterações no modo de comer só. A percepção da imagem do próprio corpo sofre influência de fatores físicos, psíquicos, econômicos, sociais e culturais. Em idosos, a diminuição da "funcionalidade" do corpo pode ser um fator explicativo para a insatisfação com a imagem corporal. ${ }^{7}$ No entanto, o valor do IMC, principalmente acima do parâmetro adequado, está relacionado comumente a altos níveis de insatisfação com o corpo em mulheres. ${ }^{2,9}$ Achado semelhante foi encontrado entre as idosas deste estudo.

As idosas entrevistadas com IMC elevado tendem a ser mais insatisfeitas com sua imagem corporal e essa insatisfação está relacionada ao excesso de peso, assim como o exposto por Tribess et al. ${ }^{10} \mathrm{~A}$ imagem corporal idealizada pelos indivíduos do presente estudo foi diferente da imagem considerada real, normalmente em escala numérica superior. Quase metade das entrevistadas almejava ter a imagem corporal número 3 da escala deStunkardetal. ${ }^{14}$ Mas 62,0\% dasidosas declararam 
ter imagens 4 ou 5. Resultados semelhantes foram encontrados em outras investigações..$^{21,31}$ Além disso, $30,2 \%$ das idosas que estavam insatisfeitas pelo excesso de peso possuíam o IMC adequado em relação à idade.

As modificações físicas, próprias do envelhecimento, são contrárias às demandas da sociedade e da mídia. Há uma necessidade imposta ao corpo, de ser jovem, sem sinais de envelhecimento e ter medidas corporais perfeitas para se encaixar numa sociedade pautada na indústria da beleza e do consumo. ${ }^{5}$ Para isso, esforços não são medidos: dietas radicais, medicamentos, procedimentos cirúrgicos e estéticos, além de exercícios demasiados são algumas das tentativas das idosas para se (re) encaixarem na sociedade.

Muitas idosas percebem seus corpos como estruturas frágeis e modificadas, denotando vivências negativas. O corpo seria uma espécie de veículo que denuncia os limites e o passar dos anos, produzindo angústias e dores (físicas e mentais). Isso independe de sua condição socioeconômica, enquadrando-se num plano mais simbólico. A aparência de um corpo mais desgastado, retocado e comprometido funcionalmente, muitas vezes não sincronizado com a mente, imputa maior dificuldade de aceitação por parte das idosas. ${ }^{3}$

As partes do corpo que as idosas do curso Alimentação, Nutrição e Terceira Idade da UnATI/UERJ menos gostavam eram os membros inferiores e abdômen. Ao contrário de alguns estudos, a parte do corpo de que mais gostavam era face, especialmente os olhos.? Gondim et al. ${ }^{31}$ evidenciaram que entre idosas praticantes de atividade física, que tendem a ter melhor percepção da imagem corporal, há a busca pela cintura fina, pelo "abdômen reto", além de pernas, glúteos e seios rígidos.

A distribuição de gordura abdominal e visceral em mulheres com mais idade tende a ser diferente, quando comparadas à fase adulta. O processo de envelhecimento é associado a um maior acúmulo de gordura visceral ou subcutânea, ocasionando aumento de massa corporal até determinada faixa de idade. Há uma redistribuição de gordura corporal, associada a mudanças metabólicas. ${ }^{32}$

Os resultados mostram que a autoclassificação do corpo real é proporcional ao valor do IMC; ou seja, quanto maior o IMC, maior a classificação numérica da imagem corporal na escala. No entanto, quanto maior a proporção corporal real, de acordo com o IMC, menor é o valor numérico do corpo idealizado na escala. A autopercepção da imagem corporal de idosas pode sofrer distorções, principalmente influenciado pelo aspecto negativo estigmatizado pela sociedade que um IMC elevado representa. Pruis \& Janowsky ${ }^{2}$ relatam que, em mulheres mais velhas, a influência da sociedade na imagem corporal pode ser maior que o próprio tamanho do corpo. Além disso, trata-se de um corpo naturalmente envelhecido, favorecendo sentimentos negativos e aumento da insatisfação.

Segundo Goffman,,$^{33}$ estigma são atributos que colocam o indivíduo em posição de "desvantagem" em relação aos demais. O autor estudou como o estigma se relaciona à construção de identidades e como os indivíduos lidam/ aceitam a condição de estigmatizado. Assim, o corpo gordo/envelhecido vai tendo sua identidade e imagem deteriorada, sendo necessárias práticas que produzam o encobrimento, numa tentativa de "encaixar-se" no padrão atual.

Vale ressaltar que o uso de desenhos de silhuetas para a autoavaliação da imagem corporal em idosos deve ser cauteloso, face às limitações relacionadas principalmente a escala dos desenhos. ${ }^{34}$ No entanto, essa ferramenta é amplamente empregada e importante na estimativa do estado nutricional e demandas internas das idosas. ${ }^{10,34}$

Como limitações, ressaltamos que os dados do presente estudo são relacionados a um grupo específico, ativo e com relativa homogeneidade. Fatores relacionados à saúde e a outras condições socioeconômicas não exploradas podem estar relacionadas à insatisfação da imagem corporal. 


\section{CONCLUSÕES}

No contexto crescente de idosos na sociedade, considerados parcela significativa do atual mercado de consumo de bens e serviços, entender aspectos relacionados à percepção do corpo nesse grupo se torna fundamental.

A prevalência de excesso de peso atinge boa parte das idosas do curso Alimentação, Nutrição e Terceira Idade da UnATI/UERJ, assim como a insatisfação coporal. Por sua vez, o envelhecimento tem sido ressaltado como fator negativo para a aparência física, e a sociedade influencia o modo como uma idosa percebe seu próprio corpo. Invariavelmente, o estado nutricional apresenta grande impacto na satisfação com o corpo, sendo a imagem corporal autopercebida pelas idosas positivamente relacionada ao IMC.

Por outro lado, a imagem corporal tida como "ideal" apresenta relação inversa, em que as idosas que apresentam IMC elevado são as que mais almejam alcançar os padrões

\section{REFERÊNCIAS}

1. Veras R. Envelhecimento populacional contemporâneo: demandas, desafios e inovações. Rev Saúde Pública 2009;43(3):548-54.

2. Pruis TA, Janowsky JS. Assessment of Body Image in Younger and Older Women. J General Psychol 2010;137(3):225-38.

3. Fernandes MGM, Garcia LG. O corpo envelhecido: percepção e vivência de mulheres idosas. Interface Comun Saúde Educ 2010;14(35):879-90.

4. Perissinotto E, Pisent C, Sergi G, Grigoletto F, Enzi G. Anthropometric measurements in the elderly: age and gender differences. Br J Nutr 2002;87(2):177-86.

5. Le Breton, D. Antropologia do corpo e modernidade. Petrópolis (RJ): Vozes; 2011.

6. Debert GG. Velhice e tecnologias do rejuvenescimento. In: Goldenberg M, organizador. Corpo, envelhecimento e felicidade. Rio de Janeiro: Civilização Brasileira; 2011. p. 65-82.

7. Damasceno VO, Vianna VRA, Vianna JM, Lacio M, Lima JRP, Novaes JS. Imagem corporal e corpo ideal. Rev Bras Ciênc Mov 2006;14(1):87-96. de um corpo magro, expressando um desejo intrínseco de se encaixar às demandas de beleza da vida contemporânea. Tais demandas vão de encontro ao perfil ilustrado até pouco tempo pela sociedade, em que uma idosa perceberia seu corpo com total conformação perante a idade cronológica.

Os resultados apontam para a necessidade de mais estudos nessa área, a fim de melhor compreender o processo de envelhecimento e de autopercepção corporal. Investigações de ordem quantitativas e qualitativas seriam de suma importância para a análise das diversas dimensões que o corpo envelhecido pode conotar e das práticas cotidianas que envolvem o cuidado com esse corpo e com a alimentação.

O entendimento de aspectos relacionados à satisfação e insatisfação corporal nos idosos é fundamental para a elaboração e implementação de intervenções direcionadas para esse grupo, reconstruindo, refletindo e problematizando a relação entre corpo, alimentação, saúde e felicidade.

8. Thomas HM, Irwin JD. What Is a Healthy Body Weight? Can J Diet Pract Res 2009;70(3):110-6.

9. Algars M, Santtila P, Varjonen M, Witting K, Johansson A, Jern P, et al. The adult body: how age, gender, and body mass index are related to body image. J Aging Health 2009;2(8):1112-32.

10. Tribess S, Virtuoso Junior JS, Petrski ED. Estado nutricional e percepção da imagem corporal de mulheres idosas residentes no nordeste do Brasil. Ciênc Saúde Coletiva 2010;15(1):31-8.

11. Menezes MFG, Santos DM, Tavares EL, Fernandes LP, Santos MS, Trindade PL. Metodologia participativa com idosos: experiência do curso nutrição e terceira idade. In: Araújo Filho T, Thiollent MJ, editores. $6^{\circ}$ Seminário de Metodologias para Projetos de Extensão - SEMPE; 2008; São Paulo, São Carlos: Cubo Multimídia/ Universidade Federal de São Carlos; 2008. p. 359-68.

12. Lohman TG, Roche AF, Martorell R. Anthropometric Standardization Reference Manual. Champaign, Illinois: Human Kinetics; 1988. 
13. Lipschitz DA. Screening for nutritional status in the elderly. Prim Care. 1994 Mar;21(1):55-67.

14. Stunkard AJ, Sorenson T, Schulsinger F. Use of the Danish Adoption Register for the study of obesity and thinness. In: Kety SS, Rowland LP, Sidman RL, Matthysse SW, editores. The genetics of neurological and psychiatric disorders. New York: Raven Press; 1983. p.115-20.

15. Instituto Brasileiro de Geografia e Estatística. Pesquisa de Orçamentos Familiares 2008-2009 Antropometria e Estado Nutricional de Crianças, Adolescentes e Adultos no Brasil. Rio de Janeiro: IBGE; 2010.

16. Brasil. Ministério da Saúde. Secretaria de Vigilância em Saúde, Departamento de Vigilância de Doenças e Agravos não Transmissíveis e Promoção de Saúde. Vigitel Brasil 2012: vigilância de fatores de risco e proteção para doenças crônicas por inquérito telefônico. Brasília : Ministério da Saúde; 2013.

17. Silveira EA, Kac G, Barbosa LS. Prevalência e fatores associados à obesidade em idosos residentes em Pelotas, Rio Grande do Sul, Brasil: classificação da obesidade segundo dois pontos de corte do índice de massa corporal. Cad Saúde Pública 2009;25(7):1569-77.

18. Barreto SM, Passos VMA, Lima-Costa MFF. Obesity and underweight among Brazilian elderly. The Bambuí health and aging study. Cad Saúde Pública 2003;19(2):605-12.

19. Goldenberg M. O corpo como capital. In: Goldenberg M, organizador. O corpo como capital: gênero, sexualidade e a moda. 2. ed. São Paulo: Estação das Letras e Cores; 2010. p. 9-16.

20. Ramos, M. S. O corpo como capital e a felicidade. In: Goldenberg M, organizador. O corpo como capital: gênero, sexualidade e a moda. 2. ed. São Paulo: Estação das Letras e Cores; 2010. p.19-28.

21. Pereira EF, Teixeira CS, Borgatto AF, Daronco LSE. Relação entre diferentes indicadores antropométricos e a percepção da imagem corporal em idosas ativas. Rev Psiquiatr Clín 2009;36(2):54-9.

22. Schieman S, Pudrovska T, Eccles R. Perceptions of body weight among older adults: analyses of the intersection of gender, race, and socioeconomic status. J Gerontol Ser B Psychol Sci Soc Sci 2007;62(6):S415-23.
23. Braggion GF. Satisfação com a aparência corporal, nível de atividade física, valor calórico da dieta e estado nutricional de mulheres com 50 anos e mais de acordo com o grupo etário [Dissertação]. São Paulo (SP): Universidade de São Paulo, Faculdade de Saúde Pública; 2002.

24. McLaren L, Kuh D. Body Dissatisfaction in Midlife Women. J Women Aging 2004;16(1-2):35-54.

25. Instituto Brasileiro de Geografia e Estatística. Síntese de Indicadores Sociais : uma análise das condições de vida da população brasileira. Rio de Janeiro: IBGE; 2010. (Estudos e Pesquisas, Informação Demográfica e Socioeconômica, n. 27).

26. Lima-Costa MFF, Matos DL, Camargos VP, Macinko J. Tendências em dez anos das condições de saúde de idosos brasileiros: evidências da Pesquisa Nacional por Amostra de Domicílios (1998, 2003, 2008). Ciênc Saúde Coletiva 2011;16(9):3689-96.

27. Harris SM. Family, Self, And Sociocultural Contributions To Body-Image Attitudes Of AfricanAmerican Women. Psychol Women Q 1995;19(1):129-45.

28. Morais FFD, Souza VBA. Factors associated with the successful aging of the socially-active elderly in the metropolitan region of Porto Alegre. Rev Bras Psiquiatr 2005;27(4):302-8.

29. Lewis DM, Cachelin FM. Body Image, body dissatisfaction, and eating attitudes in midlife and elderly women pages. Eat Disord 2001;9(1):29-39.

30. Hernández JC, Arnáiz MG. Alimentación y edad. In: Hernández JC, Arnáiz MG, orgnanizadores. Alimentación y cultura: perspectivas antropológicas. Barcelona: Ariel; 2005. p .290-313.

31. Gondim MR, Cunha SFS, Souza SG, Schmidt A, Barros DD. Percepção da imagem corporal de idosas praticantes de um programa de hidroginástica. EFDeportes 2011;15(153):1-1.

32. Zamboni M, Armellini F, Harris T, Turcato E, Micciolo R, Bergamo-Andreis IA, et al. Effects of age on body fat distribution and cardiovascular risk factors in women. Am J Clin Nutr 1997;66(1):11-5.

33. Goffman, E. Estigma: notas sobre a manipulação da identidade deteriorada. São Paulo: LTC; 1988.

34. Gardner RM, Friedman BN, Jackson NA. Methodological concerns when using silhouettes to measure body image. Percept Mot Skills 1998;86(2):387-95. 